\section{SciTeMed}

Publishing Group
Preprint Archives of Clinical Images \& Videos

\title{
Transcanal Endoscopic Ear Surgery for Congenital Cholesteatoma
}

\section{Wan-Hsuan Sun MD'; Chin-Lung Kuo, MD, PhD²; Tzu-Chin Huang, MD, MSc ${ }^{1 *}$}

${ }^{1}$ Department of Otolaryngology-Head and Neck Surgery, Cathay General Hospital, Taipei, Taiwan

${ }^{2}$ Department of Otolaryngology, Taoyuan Armed Forces General Hospital, Taoyuan, Taiwan
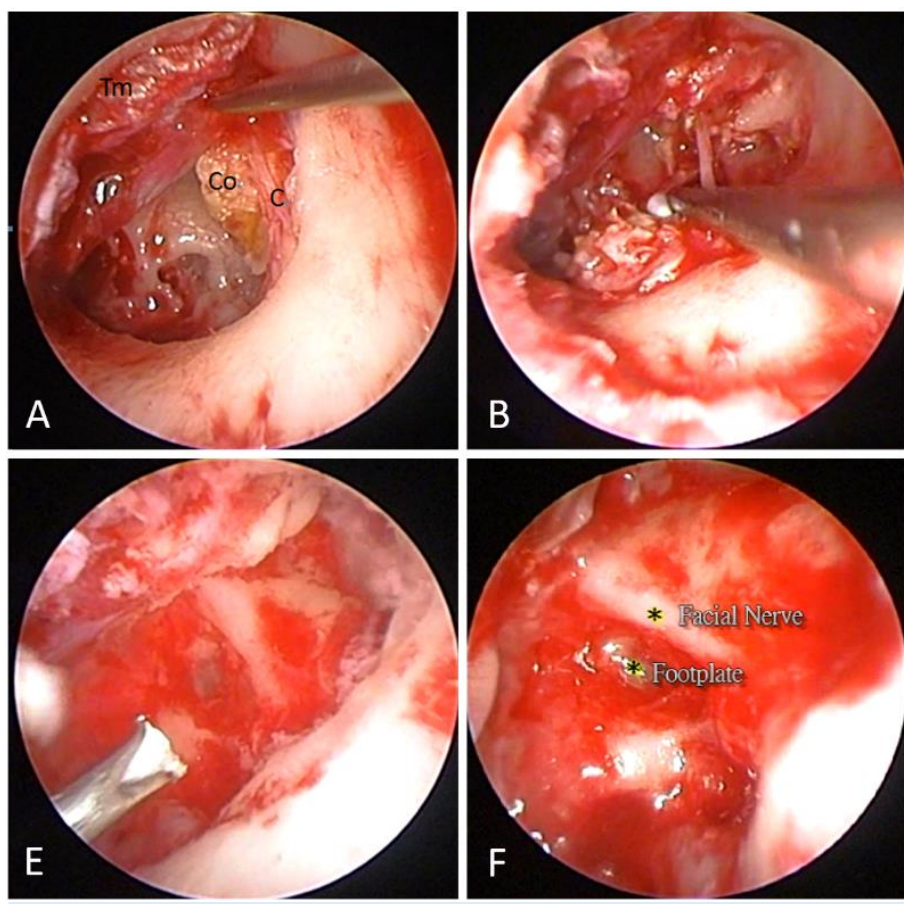
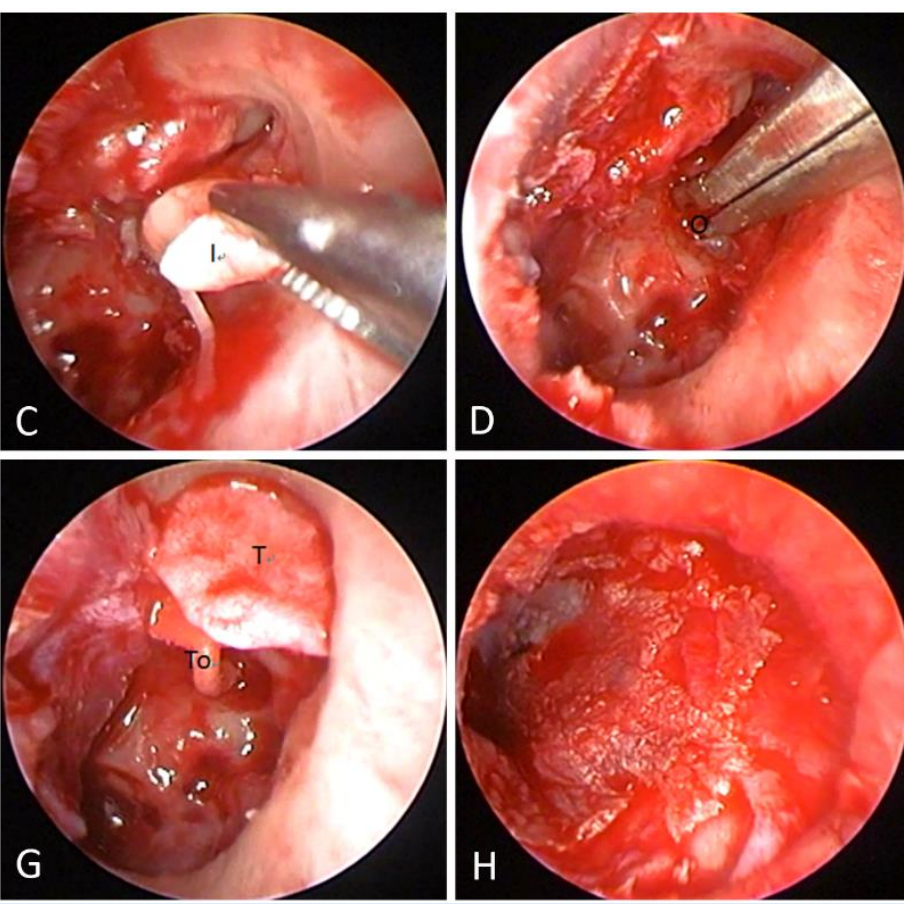

\begin{abstract}
$\Lambda$
5-year-old girl presented with a hearing impairment on the left side. She had no history of otitis media in both ears. An otoscopic examination of the left ear showed a whitish mass behind an intact tympanic membrane in the posterosuperior quadrant. A hearing test of the left ear revealed a conductive hearing loss with air-bone gap of 32.5 decibels. A high-resolution computed tomography of temporal bone revealed a soft tissue mass in the attic and posterosuperior quadrant of the left middle ear. Transcanal Endoscopic Ear Surgery (TEES) and a subsequent histopathology examination revealed a congenital cholesteatoma. This video demonstrates how to remove a congenital cholesteatoma using TEES. (A) The tympano-meatal flap was elevated, and cholesteatoma was identified. (B) After drilling the scutum, cholesteatoma was removed. (C) The incus was removed. (D) Residual cholesteatoma was removed. (E) Water irrigation of the middle ear. (F) The footplate and facial nerve were identified. (G) Scutum reconstruction by tragus cartilage and ossicle chain reconstruction by Total Ossicular Replacement Prosthesis (TORP). (H) Tympano-meatal flap was replaced. Abbreviations: $\mathrm{C}$ = cholesteatoma; $\mathrm{Co}=$ corda tympani nerve; $\mathrm{I}=$ incus; $\mathrm{O}=$ oval window; $\mathrm{T}=$ tragus cartilage; $\mathrm{To}=\mathrm{TORP} ; \mathrm{Tm}=$ tympano-meatal flap.

DOI: 10.24983/scitemed.paciv.2017.00015
\end{abstract}

*Correspondence: Tzu-Chin Huang, MD, MSc

Department of Otolaryngology-Head and Neck Surgery, Cathay General Hospital, Taipei, Taiwan. E-mail: tzuchin_h@yahoo.com.tw

Received: May 13, 2017; Accepted: May 26, 2017; Published: May 26, 2017

Preprint Archives of Clinical Images \& Videos. 2017;1(2):3 DOI: $10.24983 /$ scitemed.paciv.2017.00015

Copyright (C) 2017 The Author(s). This is an open-access article distributed under the terms of the Creative Commons Attribution 4.0 International License (CC-BY).

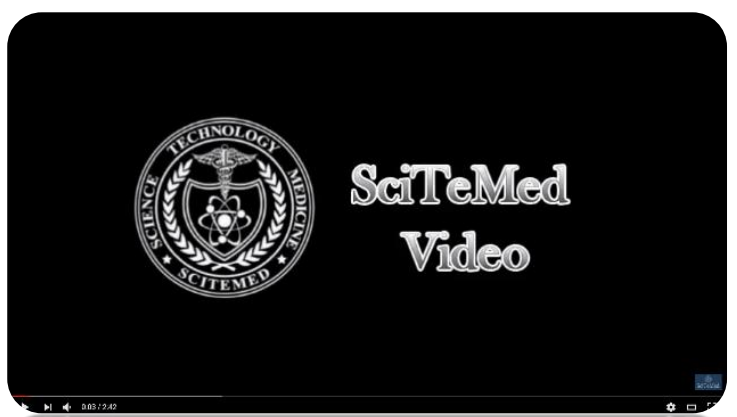

Video Link 